\title{
Histological antiphospholipid-associated
} nephropathy versus lupus nephritis in patients with systemic lupus erythematosus:

\section{an observational cross-sectional study with longitudinal follow-up}

Jakob Gerhardsson ${ }^{1 *}$, Birgitta Sundelin², Agneta Zickert ${ }^{1}$, Leonid Padyukov', Elisabet Svenungsson ${ }^{1}$ and Iva Gunnarsson ${ }^{1}$

\begin{abstract}
Introduction: Renal involvement is a severe complication in systemic lupus erythematosus (SLE). Moreover, a subset of SLE patients develop the anti-phospholipid syndrome (APS), characterised by the occurrence of anti-phospholipid antibodies in combination with macro- and microvascular thrombotic manifestations, including acute and chronic antiphospholipid-associated nephropathy (APLN). Clinical presentations of lupus nephritis and APLN are similar and a renal biopsy is necessary to differentiate between the conditions. Our aim with this study was to investigate the occurrence of histopathological findings consistent with APLN (hAPLN) in renal biopsies from SLE patients and to investigate associations with anti-phospholipid antibody specificities, clinical manifestations, HLA-DRB1 alleles, and long-term renal outcome.
\end{abstract}

Method: Consecutive renal biopsies from 112 SLE patients with renal involvement were investigated and evaluated for findings of hAPLN; in all there were 236 renal biopsies. Data from biopsy reports and clinical information were collected. Autoantibodies against cardiolipin and $\beta_{2}$-glycoprotein-1 were measured by enzyme-linked immunosorbent assay. A lupus anticoagulant test was determined with a modified Dilute Russel Viper Venom method. HLA genotyping was performed by sequence-specific primer PCR. Renal outcome was determined at study end.

Results: The prevalence of hAPLN was $14.3 \%$ among SLE patients with renal involvement. Compared to patients with pure lupus nephritis, occurrence of hAPLN was associated with intima changes (odds ratio $(O R)=24 ; 95 \%$ confidence interval $(\mathrm{Cl}), 3.0$ to $189.8 ; P<0.0001)$, hypertensive vascular changes $(\mathrm{OR}=7.8 ; 95 \% \mathrm{Cl}, 1.6$ to 39.4 ; $P=0.01)$, inflammatory infiltrates $(\mathrm{OR}=6.5 ; 95 \% \mathrm{Cl}, 1.7$ to $25.1 ; P=0.007)$ and tubular atrophy $(\mathrm{OR}=13.1 ; 95 \% \mathrm{Cl}$, 1.7 to $103.6 ; P=0.002)$. hAPLN was associated with the presence of cardiolipin antibodies $(\mathrm{OR}=3.3 ; 95 \% \mathrm{Cl}, 1.0$ to $10.8 ; P=0.05)$ and triple anti-phospholipid antibody positivity $(\mathrm{OR}=4.2 ; 95 \% \mathrm{Cl}, 1.3$ to $13.7 ; P=0.02)$. Patients with hAPLN were more hypertensive $(\mathrm{OR}=3.8 ; 95 \% \mathrm{Cl}, 1.2$ to $12.3 ; P=0.03)$ and had higher levels of creatinine as compared to lupus nephritis patients (median 116 versus $75 \mu \mathrm{mol} / \mathrm{L} ; P<0.0001$ ). We found significantly higher frequency of HLA-DRB $1 * 13(\mathrm{OR}=5.1 ; 95 \% \mathrm{Cl}, 1.7$ to $15.4 ; P=0.03)$ and development of end-stage renal disease $(\mathrm{OR}=5.8 ; 95 \% \mathrm{Cl}, 1.7$ to $19.7 ; P=0.008)$ in $\mathrm{hAPLN}$ compared with lupus nephritis.

\footnotetext{
* Correspondence: jakob.gerhardsson@ki.se

'Department of Medicine, Unit of Rheumatology, Karolinska University

Hospital, Karolinska Institutet, S-171 76 Stockholm, Sweden

Full list of author information is available at the end of the article
} 
(Continued from previous page)

Conclusion: hAPLN is a severe and often unrecognized condition in SLE patients with renal involvement. We have demonstrated an increased risk for development of renal impairment and a genetic predisposition in hAPLN patients compared to lupus nephritis patients.

\section{Introduction}

Systemic lupus erythematosus (SLE) is a systemic autoimmune disease characterized by a heterogeneous set of clinical presentations including renal manifestations and the presence of autoantibodies. Antiphospholipid antibodies (aPL) are detected in 30 to $50 \%$ of patients with SLE and are associated with thromboembolic complications. Lupus nephritis (LN) of varying severity affects up to $60 \%$ of SLE patients [1,2]. Apart from the glomerular lesions seen in LN, patients with SLE may develop renal microangiopathy which may be identical to findings seen in patients with the antiphospholipid syndrome (APS) [3]. The microangiopathy can be acute or chronic and may occur in isolation or with concurrent LN [4-7]. Of note, the clinical presentations are similar and a renal biopsy is required to distinguish APS-related findings from pure LN.

In the most recent consensus criteria of APS [3], the term antiphospholipid-associated nephropathy (APLN) was suggested to describe the entity of aPL in association with renal vasculopathy. In previous publications, however, various definitions have been used to describe the acute condition thrombotic microangiopathy (TMA) [7], isolated renal microthrombi [5], or both acute and chronic renal findings $[4,6]$. Thus, full comparisons between studies on renal vasculopathy in SLE populations are difficult to perform. It is also of note that aPL have not been identified in all cases with APLN in SLE $[4,7]$ and the natural history of APLN development over time is largely unknown.

aPL are a family of autoantibodies which target membrane structures such as phospholipids or phospholipidbinding proteins. In clinical practice, aPL positivity is determined by occurrence of anticardiolipin antibodies $(\mathrm{aCL})$ or of those to the protein co-factor $\beta_{2}$-glycoprotein-1 $\left(\beta_{2} \mathrm{GP} 1\right)$ as measured by enzyme-linked immunosorbent assay (ELISA), or positivity in the functional lupus anticoagulant (LA) test. LA detects aPL, which are capable of prolonging in vitro coagulation assays $[3,8,9]$, and has been shown to be more closely related to clinical symptoms than positive ELISA tests [1,10]. With regard to APLN, data have been divergent concerning the associations with different aPL [4-7].

There is a genetic predisposition to certain subphenotypes of SLE, including nephritis $[11,12]$. Furthermore, variations in human leukocyte antigen (HLA) genes have previously been found to associate with aPL in SLE [13]. We have recently demonstrated an association between the development of both aPL and macrovascular thrombotic events among SLE patients with the HLA-DRB1 alleles and with the polymorphism in the signal transducer and activator of transcription factor 4 (STAT4) gene $[12,14]$. The STAT4 risk allele on chromosome 2 was also recently demonstrated to be associated with LN as well as with the development of severe renal insufficiency, thus further indicating that genetic predisposition is of importance for disease progression in LN [11]. No genetic studies on APLN were known at the time of our investigation.

We have investigated the occurrence of histopathological renal microangiopathy in accordance with APLN in consecutive biopsies from a large cohort of SLE patients with renal involvement. We chose to define the renal vascular findings, regardless of antibody findings, as histopathological APLN (hAPLN). We studied the renal outcome in patients with hAPLN in comparison to patients with pure LN and investigated the occurrence of hAPLN over time in repeated renal biopsies. Although hAPLN has been associated with more severe renal disease in SLE [4-6], data on long-term outcome are still an issue of concern and were therefore investigated in this study. Finally, we studied the impact of HLA-DRB1 alleles on the occurrence of hAPLN.

\section{Methods \\ Patients}

Consecutive renal biopsies performed between 1995 and 2011 in patients included in the Karolinska SLE Cohort [12] were used in this study. All patients fulfilled at least four classification criteria for SLE [15] and were recruited from both the rheumatology and nephrology units in order to obtain a representative selection with regard to renal involvement. Information was collected from first study biopsy until 31 December 2012. Patients with renal findings that were not related to LN or hAPLN were not included in the study. All patients had given informed written consent to participate in the study and the study protocol was reviewed and approved by the regional ethics committee at Karolinska Institutet in Stockholm, Sweden.

\section{Renal biopsies and clinical information}

All renal biopsies were evaluated for findings in accordance with hAPLN and/or LN. Simultaneously with the biopsies, clinical and laboratory data including occurrence 
of hypertension, treatment with corticosteroids, anticoagulants, and antihypertensive medication were collected.

Hypertension was considered as present if patients were diagnosed with and prescribed medications for high blood pressure or if the patient had a blood pressure $>140 / 90$ $\mathrm{mmHg}$ at time of biopsy. Patients treated with angiotensin converting enzyme inhibitors and/or angiotensin receptor blockers for anti-proteinuric indications only were not regarded as hypertensive.

\section{Evaluation of renal biopsies}

All biopsies were evaluated by one experienced pathologist (BS) and examined with light microscopy, immunofluorescence and electron microscopy. Glomerular lesions were assessed according to World Health Organization criteria [16] and/or the International Society of Nephrology/Renal Pathology Society criteria [17] depending on the date of biopsy. Information about vascular findings, intima change, interstitial fibrosis, inflammatory infiltrates, tubular atrophy and hypertonic vascular changes as well as findings from electron microscopy were also extracted from the pathology reports.

Vascular findings consistent with APLN [3] were reported and further divided into acute TMA and chronic hAPLN. TMA was defined as occurrence of fibrin thrombi in arterioles and/or glomerular capillaries. Definition of chronic features of hAPLN included presence of fibrous intimal hyperplasia with or without recanalization, fibrous and/or fibrocellular occlusions of arteries and arterioles and focal cortical atrophy $[4,6,18]$. In cases where acute TMA occurred without concomitant LN, other conditions that may cause endothelial dysfunction were excluded before inclusion in the study. Cases in which a definite diagnosis of hAPLN was doubtful were re-assessed by the pathologist for a definite diagnosis.

\section{Laboratory data}

Laboratory data were analysed at the time of the biopsy and performed according to clinical routine. In this study we included levels of creatinine $(\mu \mathrm{mol} / \mathrm{L})$, albumin $(\mathrm{g} / \mathrm{L})$ and haemoglobin $(\mathrm{g} / \mathrm{L})$.

\section{Autoantibodies}

Autoantibodies were analyzed in the Department of Clinical Immunology at the Karolinska University Hospital. For $\mathrm{aCL}$ and anti- $\beta_{2} \mathrm{GP} 1$ antibody detection, ELISA and multiplex methods were used. aCL was evaluated for both immunoglobulin (Ig)G and IgM isotypes. For anti- $\beta_{2} \mathrm{GP} 1$, only the IgG isotype was measured. We also recorded if patients had a positive LA, determined with a modified Dilute Russel Viper Venom method (Biopool, Umeå, Sweden).

Being a retrospective observational study (spanning January 1995 to December 2012), methods for autoantibody detection have changed during the study period.
Tabulated data were thus recorded as positive or negative according to the cut-off levels of the routine method used at the time of analysis.

The data available in the Karolinska SLE Cohort included current and retrospective information regarding serological profile and occurrence of aCL and anti- $\beta_{2}$ GP1 , as well as LA positivity. Autoantibody and LA positivity was also determined at the time of renal biopsy. Autoantibody and/or LA positivity was thus determined both at and before biopsy, which enables a confirmation of autoantibody findings as required to fulfil the classification criteria for definite APS according to Miyakis and colleagues [3]. Furthermore, we determined if the patients were ever positive in all three aPL tests (aCL (IgM and IgG), anti$\beta 2 \mathrm{gGP}-1$ (IgG) and LA). This combination is described by Pengo and colleagues as triple positivity [19], and is associated with a more severe prognosis.

\section{Long-term follow-up}

The renal function was estimated at last follow-up by the Modified Diet in Renal Disease formula [20], and the patients were classified into chronic kidney disease classes 1 to 5 [21]. We defined the time the patient reached chronic kidney disease class 5 as end-stage renal disease (ESRD).

\section{HLA typing}

Two-digit HLA-DRB1 genotyping for the HLA-DRB1gene was previously performed by sequence-specific primer PCR and was also used for the present study [12]. We studied the following HLA-DRB1 allelic groups: DRB1*01, DRB1*03, DRB1*04, DRB1*07, DRB1*08, DRB 1*09, DRB1*10, DRB1*11, DRB1*12, DRB1*13, DRB1*14, DRB1*15 and DRB1"16 using dominant model.

\section{Statistics}

Chi-squared test and Fisher's exact test were used as appropriate for statistical analysis of selected categorical variables, which included vascular changes consistent with hAPLN, presence of aCL, anti- $\beta_{2} \mathrm{GP} 1$ autoantibodies, positive tests for LA, and hypertension. Odds ratios (ORs) were calculated from contingency tables assessing the risk of co-appearance of selected variables. For variables with statistical significance, 95\% confidence intervals (CIs) were calculated.

Skewed continuous variables were log transformed to obtain a normal distribution. For quantitative variables, such as creatinine, albumin and haemoglobin, analysis of variance was used. Mean and extremes are given for descriptive purposes. Bonferroni correction was performed for multiple comparisons when tabulating HLA genotypes.

For statistical analysis JMP-11 software (SAS institute, NC, USA) was used. Statistical significance was accepted at $P<0.05$. 


\section{Results}

\section{General patient characteristics}

The original study cohort consisted of 126 patients with a diagnosis of SLE who had renal biopsy performed due to signs of renal involvement. Fourteen patients were excluded from the study due to histopathological findings other than LN or hAPLN (seven with focal necrotizing vasculitis in the active or inactive phase, one with IgA nephropathy, one with thin basal membranes, one with tubulointerstitial nephritis in accordance with Sjögren's syndrome, and four with unspecific chronic lesions with no prior history/biopsy with LN). The final study cohort thus consisted of 112 patients who had been subject to 236 biopsies in total. The median number of biopsies per patient was 2 (range 1 to 7 ) and the median age at first study biopsy was 38 years (range 18 to 84 years).

There were 89 females (79.5\%) and 23 males (20.5\%). In all, 201 biopsies (85.2\%) were from female patients and 35 (14.8\%) from male participants. Patient characteristics are displayed in Table 1.

\section{Renal biopsies}

Overall, 16/112 patients (14.3\%) had findings in accordance with hAPLN in at least one biopsy. Among all biopsies, hAPLN was detected in 20/236 (8.5\%). Thus, four patients had two biopsies with hAPLN findings. Ten biopsies revealed findings of LN with concomitant findings of hAPLN while 10 had pure hAPLN. Of 236 biopsies, 226 (95.8\%) had changes compatible with $\mathrm{LN}$ according to either the World Health Organization [16] or International Society of Nephrology/Renal Pathology Society criteria [17].

Of the sixteen hAPLN patients, seven (43.8\%) had findings in their first study biopsy while 9/16 (56.2\%) patients developed hAPLN following an initial diagnosis of LN (median 8.3 years, range 1 to 28 years) from first biopsy. Conversely, in three cases hAPLN was seen in the first study biopsy, but not in a repeated biopsy. In the hAPLN group, 4/20 (20\%) biopsies were consistent with the acute setting (TMA), while the majority $16 / 20$ (80\%) had a more chronic phase of hAPLN.

For statistical purposes, when comparing clinical/laboratory variables in the $\mathrm{LN}$ group $(\mathrm{n}=96)$ versus the hAPLN

Table 1 Clinical characteristics in all patients at first study biopsy

\begin{tabular}{|c|c|c|c|c|}
\hline & $\begin{array}{l}\text { All patients } \\
(n=112)\end{array}$ & $\begin{array}{l}\text { LN } \\
(n=96)\end{array}$ & $\begin{array}{l}\text { hAPLN* } \\
(n=16)\end{array}$ & $P$ value \\
\hline Median age (years (range)) & $38(18-84)$ & $37(18-84)$ & $45(26-62)$ & 0.37 \\
\hline Male/female & $23 / 89$ & $18 / 78$ & $5 / 11$ & 0.3 \\
\hline Serum creatinine ( $\mu \mathrm{mol} / \mathrm{L}$; median) & 77 & 75 & 116 & $<0.0001$ \\
\hline Serum albumin (g/L; median) & 29 & 29 & 30 & 0.9 \\
\hline Haemoglobin (g/L; median) & 113 & 114 & 110 & 0.2 \\
\hline Hypertension (n (\%)) & $41(37)$ & $30(31)$ & $11(69)$ & 0.03 \\
\hline Systolic blood pressure (mmHg; median) & 125 & 120 & 137 & 0.01 \\
\hline Diastolic blood pressure (mmHg; median) & 80 & 80 & 80 & 0.1 \\
\hline \multicolumn{5}{|l|}{ Autoantibodies (n (\%))* } \\
\hline $\mathrm{aCL}$ & $58(52)$ & $46(48)$ & $12(75)$ & 0.05 \\
\hline anti- $\beta_{2} \mathrm{GP1}$ & $23(21)$ & $17(18)$ & $6(38)$ & 0.07 \\
\hline LA & $28(25)$ & $21(22)$ & $7(44)$ & 0.06 \\
\hline Single positive aPL & $36(32)$ & $31(32)$ & $5(31)$ & 0.9 \\
\hline Double positive aPL & $9(8)$ & $8(8)$ & $1(6)$ & 0.8 \\
\hline Triple positive aPL & $18(16)$ & $12(13)$ & $6(38)$ & 0.02 \\
\hline Definite APS & $13(12)$ & $10(10)$ & $3(19)$ & 0.3 \\
\hline \multicolumn{5}{|l|}{ Treatment (n (\%)) } \\
\hline Prednisolone (mg/day; median (range)) & $8(0-60)$ & $8(0-40)$ & $15(0-60)$ & 0.006 \\
\hline ACEi/ARB & $40(36)$ & $29(30)$ & $11(69)$ & 0.007 \\
\hline Warfarin & $10(9)$ & $7(7)$ & $3(19)$ & 0.2 \\
\hline ASA & $16(14)$ & $13(14)$ & $3(19)$ & 0.7 \\
\hline
\end{tabular}

*Previous findings and/or at renal biopsy. $P$ values are the comparison between the two diagnostic groups (LN/hAPLN). Values in bold are significant at $<0.05$. Single positive, double positive and triple positive refers to autoantibody findings at or before biopsy. ACEi, angiotensin converting enzyme inhibitor; aCL, anticardiopin antibodies; anti- $\beta_{2}$ GP1, anti- $\beta_{2}$-glycoprotein-1; APS, antiphospholipid syndrome; ARB, angiotensin receptor blocker; ASA, acetylsalicylic acid; hAPLN, histopathological antiphospholipid-associated nephropathy; LA, lupus anticoagulant; LN, lupus nephritis. 
group ( $\mathrm{n}=16$ ), we used data collected at the first study biopsy in the LN patients and the first biopsy demonstrating hAPLN findings in the hAPLN group. Thus, nine patients with initial LN were included in the LN group and data from the first hAPLN biopsy was used for comparison between the groups. No association between age or gender comparing the LN and hAPLN groups was found. Data on all biopsy findings in the hAPLN patients are shown in Table 2.

\section{Histopathology}

We extracted the histopathological findings available in biopsy reports from all patients. Compared to the LN group, hAPLN patients were associated with occurrence of hypertensive vascular changes $(P=0.01)$, inflammatory infiltrates $(P=0.007)$, interstitial fibrosis $(P=0.02)$, intima changes $(P<0.0001)$, and tubular atrophy $(P=0.002)$.

We found that all hAPLN biopsies without LN findings $(n=10)$ had inflammatory changes. In contrast, of the patients with hAPLN and concomitant LN 5 had inflammatory changes while the remaining 5 had no signs of inflammation (data not shown).

The histopathological findings are shown in Table 3.

\section{Routine laboratories}

In all 112 patients, the median serum creatinine was 77 $\mu \mathrm{mol} / \mathrm{L}$ (mean 98, range 39 to 514 ) at first study biopsy. Serum creatinine levels were higher in the hAPLN group (median 116) as compared to the LN group (median 75; $P<0.0001$ ). Albumin and haemoglobin levels did not differ between the hAPLN and LN groups as shown in Table 1.

\section{Blood pressure}

Twenty-seven patients (24.2\%) had a diagnosis of, and received treatment for, hypertension at first study biopsy. Of these, nine also had vascular changes consistent with hAPLN. There was a positive association between hAPLN and hypertension (OR $=3.8 ; 95 \% \mathrm{CI}$, 1.2 to $12.3 ; P=0.03)$.

Table 2 Histopathological classification*, age and development of end-stage renal disease in hAPLN patients

\begin{tabular}{|c|c|c|c|c|c|c|}
\hline Patient number & $\begin{array}{l}\text { Biopsies prior to } \\
\text { study start (age) }\end{array}$ & $\begin{array}{l}\text { Biopsy prior to } \\
\text { hAPLN (age) }\end{array}$ & Biopsy I (age) & Biopsy II (age) & Biopsy III (age) & ESRD (age) \\
\hline 1 & - & - & hAPLN (54) & - & - & - \\
\hline 2 & II (36) & - & hAPLN/IV (38) & ॥ (39) & $V(41)$ & $+(55)$ \\
\hline 3 & - & - & hAPLN (49) & hAPLN (53) & - & - \\
\hline 4 & hAPLN (8) & - & hAPLN/II (28) & - & - & $+(28)$ \\
\hline \multirow[t]{4}{*}{5} & - & IV (35) & hAPLN (47) & - & - & - \\
\hline & & II (36) & & & & \\
\hline & & IV (39) & & & & \\
\hline & & IV(42) & & & & \\
\hline \multirow[t]{3}{*}{6} & IV (26) & V (35) & hAPLN/N (36) & - & - & - \\
\hline & IV (28) & & & & & \\
\hline & III (29) & & & & & \\
\hline 7 & - & II (40) & hAPLN/N (43) & - & - & - \\
\hline 8 & - & $\|(27)$ & hAPLN (29) & Scarring (29) & hAPLN (34) & $+(38)$ \\
\hline 9 & - & III (25) & hAPLN/II (26) & - & - & - \\
\hline \multirow[t]{4}{*}{10} & $V(16)$ & $V(22)$ & hAPLN/N (27) & V (33) & - & - \\
\hline & & V (23) & & & & \\
\hline & & V (24) & & & & \\
\hline & & $V(26)$ & & & & \\
\hline 11 & $\|+V(24)$ & - & hAPLN/II + V (52) & - & - & - \\
\hline 12 & - & - & hAPLN/N (30) & hAPLN/N (33) & - & $+(36)$ \\
\hline 13 & - & - & hAPLN/N (58) & V (59) & - & - \\
\hline 14 & - & - & hAPLN (61) & - & - & - \\
\hline 15 & - & - & hAPLN (55) & hAPLN (58) & - & $+(58)$ \\
\hline 16 & $V(56)$ & - & hAPLN (62) & - & - & $+(66)$ \\
\hline
\end{tabular}

*Class II, III, IV and V refers to either the World Health Organization [16] or International Society of Nephrology/Renal Pathology Society [17] classification criteria of lupus nephritis, histopathological antiphospholipid-associated nephropathy (hAPLN) and end-stage renal disease (ESRD). 
Table 3 Histopathological findings in the study cohort

\begin{tabular}{|c|c|c|c|c|}
\hline & $\begin{array}{l}\text { All patients } \\
(n=112)\end{array}$ & $\begin{array}{l}\text { LN } \\
(n=96)\end{array}$ & $\begin{array}{l}\text { hAPLN } \\
(n=16)\end{array}$ & $P$ value \\
\hline $\begin{array}{l}\text { Hypertensive vascular } \\
\text { changes }\end{array}$ & $37(33)$ & $29(30)$ & $8(50)$ & 0.01 \\
\hline Inflammatory infiltrates & $41(37)$ & $30(31)$ & $11(69)$ & 0.007 \\
\hline Interstitial fibrosis & $82(73)$ & $67(70)$ & $15(94)$ & 0.02 \\
\hline Intima changes & $50(45)$ & $35(37)$ & $15(94)$ & $<0.0001$ \\
\hline Tubular atrophy & $63(56)$ & $48(50)$ & $15(94)$ & 0.002 \\
\hline
\end{tabular}

Values are shown as $\mathrm{n}(\%) . P$ values are comparisons between the two diagnostic groups: lupus nephritis (LN) and histopathological antiphospholipid-associated nephropathy (hAPLN).

The median systolic blood pressure in all patients was $125 \mathrm{mmHg}$ (range 90 to $190 \mathrm{mmHg}$ ) and the median diastolic blood pressure at biopsy was $80 \mathrm{mmHg}$ (range 55 to $110 \mathrm{mmHg}$ ). Systolic blood pressure was higher in hAPLN patients (median $137 \mathrm{mmHg}$, range 100 to 190 $\mathrm{mmHg}$ ) as compared to $\mathrm{LN}$ patients (median $120 \mathrm{mmHg}$, range 90 to $190 \mathrm{mmHg} P=0.01$ ), but no difference was found when comparing diastolic blood pressure. Results are shown in Table 1.

\section{Antiphospholipid antibodies and lupus anticoagulant}

In all patients, 58 (52\%) tested positive for aCL, 23 (21\%) for anti- $\beta_{2} \mathrm{GP1}$ and 28 (25\%) for LA at and/or prior to first study biopsy.

Of the patients in the LN group, 46 (48\%) tested positive for aCL, 17 (18\%) for anti- $\beta_{2} \mathrm{GP} 1$ and 21 (22\%) for LA.

Among the hAPLN patients, 12 (75\%) tested positive for aCL, 6 (38\%) for anti- $\beta_{2}$ GP1 and 7 (44\%) for LA. Data on antibodies and LA are presented in Table 1.

hAPLN was positively associated with the presence of aCL $(\mathrm{OR}=3.3 ; 95 \% \mathrm{CI}, 1.0$ to $10.8 ; P=0.05)$. Although the association between hAPLN and anti- $\beta_{2}$ GP1 or LA $(P=0.07$ and $P=0.06$, respectively) did not reach statistical significance, the combinations between all three tests (triple positivity, as defined by Pengo and colleagues [19]) was increased in the hAPLN patients compared to the LN group $(\mathrm{OR}=4.2 ; 95 \% \mathrm{CI}, 1.29$ to $13.65 ; P=0.02)$.

\section{Treatment at time of first renal biopsy}

Oral prednisolone was prescribed to 76 (67.8\%) patients. The median dosage in all patients at the time of biopsy was $8 \mathrm{mg} /$ day (mean $11 \mathrm{mg} /$ day, range 0 to $60 \mathrm{mg} /$ day). The median dosage in the hAPLN group was $15 \mathrm{mg} /$ day and in the LN group was $8 \mathrm{mg} / \mathrm{day}(P=0.006)$. The number of patients receiving angiotensin converting enzyme inhibitors and/or angiotensin receptor blockers, acetylsalicylic acid, and warfarin are shown in Table 1.

\section{Outcome at last follow-up}

Development of ESRD was seen in 15 patients, comprising $9 / 96(9.4 \%)$ in the LN group and 6/16 (37.5\%) in the
hAPLN group $(\mathrm{OR}=5.8 ; 95 \% \mathrm{CI}, 1.7$ to $19.7 ; P=0.008)$. The mean time to development of ESRD was 4.4 years following first study biopsy (3.4 years for LN, and 5.9 years for hAPLN).

\section{HLA-DRB1 allotypes}

Patients with hAPLN were more likely to carry the HLADRB1*13 allele in comparison to LN patients $(\mathrm{OR}=5.1$; 95\% CI, 1.7 to 15.4; $P=0.03$ after Bon Ferroni correction). There were no differences between hAPLN and LN for HLA-DRB1"01, "03, "04, "07, "08, "09, "10, "11, "12, "14, "15 or "16 allotypes. Results shown in Table 4.

\section{Discussion}

We detected renal microvascular changes in accordance with hAPLN in $14.3 \%$ of the patients in a large cohort of SLE patients with biopsy-proven renal involvement. Overall, hAPLN patients presented with more hypertension, severe histopathology and impaired renal function when compared to patients with pure LN. hAPLN was associated with aCL and with triple aPL positivity. Furthermore, the HLA-DRB1*13 allele was associated with hAPLN in the investigated patients. Our results thus indicate for the first time that a genetic predisposition may contribute to hAPLN in SLE.

In previous studies, at least one histological lesion compatible with hAPLN, either as isolated findings or in addition to LN, was seen in 24 to $32 \%$ of patients with SLE [6,7]. Zheng and colleagues [5] detected glomerular microthrombi in $20 \%$ of LN patients, which was associated with poorer renal function as well as more severe renal tissue injury. In our study, the observed prevalence of hAPLN was somewhat lower compared to previous studies. Differences in definitions, varying study populations, and indication for renal biopsy are likely to contribute to the observed differences in prevalence. Of note, our study included mainly Caucasian patients as opposed to the Asian population included in previous studies $[5,7]$ and ethical differences could also have contributed.

It has previously been proposed that outer folded or wrinkled basement membrane may be characteristic of microangiopathy in association with APS [22]. However, in the hAPLN group this was only detected in one of the patients (data not shown). Thus, electron microscopy evaluation did not seem to add diagnostic value.

We found a higher proportion of inflammatory infiltrates in the hAPLN group compared to the LN group. In cases with concomitant LN and hAPLN, it was not possible to determine whether the inflammatory infiltrates were caused by either condition. The striking finding of inflammation in the pure hAPLN group, however, strongly indicates that circulatory causes (for example, ischaemia caused by hAPLN or hypertension) have contributed to occurrence of the inflammatory infiltrates. 
Table 4 HLA-DRB1 alleles and association with hAPLN

\begin{tabular}{|c|c|c|c|c|c|}
\hline HLA-DRB1 & $\begin{array}{l}\text { All patients } \\
\left(\mathrm{n}=110^{*}\right)\end{array}$ & $\begin{array}{l}\mathrm{LN} \\
(\mathrm{n}=94)\end{array}$ & $\begin{array}{l}\text { hAPLN } \\
(n=16)\end{array}$ & $\begin{array}{l}P \text { value (after } \\
\text { Bonferroni } \\
\text { adjustment) }\end{array}$ & $\begin{array}{l}P \\
\text { value }\end{array}$ \\
\hline${ }^{*} 01$ & $16(15)$ & $16(17)$ & $0(0)$ & NS & 0.1 \\
\hline *03 & $43(39)$ & $36(38)$ & $7(44)$ & NS & 0.7 \\
\hline *04 & $23(21)$ & $20(21)$ & $3(19)$ & NS & NS \\
\hline${ }^{*} 07$ & $12(11)$ & $11(12)$ & $1(6)$ & NS & NS \\
\hline *08 & $7(6)$ & $6(6)$ & $1(6)$ & NS & NS \\
\hline *09 & $4(4)$ & $4(4)$ & $0(0)$ & NS & NS \\
\hline$* 10$ & $3(3)$ & $2(2)$ & $1(6)$ & NS & 0.4 \\
\hline$* 11$ & $11(10)$ & $11(12)$ & $0(0)$ & NS & 0.4 \\
\hline$* 12$ & $4(4)$ & $4(4)$ & $0(0)$ & NS & NS \\
\hline *13 & $28(26)$ & $19(20)$ & $9(56)$ & 0.03 & 0.002 \\
\hline *14 & $4(4)$ & $3(3)$ & $1(6)$ & NS & 0.5 \\
\hline$* 15$ & $49(45)$ & $44(47)$ & $5(31)$ & NS & 0.3 \\
\hline *16 & $3(3)$ & $2(2)$ & $1(6)$ & NS & 0.4 \\
\hline
\end{tabular}

Values are shown as $\mathrm{n}(\%) . P$ values are comparisons between the two diagnostic groups: lupus nephritis (LN) and histopathological antiphospholipid-associated nephropathy (hAPLN). NS, not significant.

${ }^{*}$ Genetic analysis had not been performed on two of the patients.

The overall lower prevalence (8.5\%) of hAPLN in all biopsies could also be due to the large number of repeated biopsies in the study cohort. However, the repeated biopsies available gave us a unique opportunity to study the natural history of hAPLN, its development and disappearance. Of note is that, in three of the patients with hAPLN, all findings had disappeared at re-biopsy. Interestingly, this implies that hAPLN can be transient, or possibly focal. Another important observation is that $56 \%$ of hAPLN appeared de novo in patients who had previously had pure LN. Taken together, these observations demonstrate that repeated biopsies are important when the clinical course indicates an unexplained deterioration of renal function, especially in patients who are aPL positive.

We found that hAPLN was associated with aCL, but a strong trend between hAPLN and anti- $\beta_{2}$ GP1 or LA was also detected. The lack of statistical significance for anti$\beta 2 \mathrm{gGP1}$ and LA was most likely due to the sample size. Consistent with our findings, Tektonidou and colleagues [4] reported an association between APLN and aCL, but also with LA. On the other hand Daugas and colleagues [6] demonstrated a strong association between APLN and LA, but not between APLN and aCL. In a study on occurrence of glomerular microthrombi, there was a strong association with LA and anti- $\beta_{2} \mathrm{GP} 1$, but not with $\mathrm{aCL}$ [5]. The divergent aPL associations previously reported are likely to be due to methodological issues, as different assays for aPL and LA detection have been used and cut-off values have varied between studies.

The strong association of hAPLN with aCL is consistent with APLN being a manifestation of APS [3]. It is, however, also important to note that $25 \%$ of patients with renal findings in accordance with hAPLN had no detectable aPL at all using the assays available at the time. Similar findings have previously been reported [4,7].

It has previously been shown that patients suffering from SLE and systemic hypertension have a worse prognosis concerning renal survival as compared to normotensive individuals [23]. Furthermore, we have previously demonstrated that aPL in SLE patients is a risk factor for cardiovascular disease [24] and both aPL and loss of renal function predicted mortality in SLE [25]. In the present study there was a clear association between hAPLN and hypertension. The hAPLN group was also associated with higher creatinine levels, which is in line with previous publications [4-6]. In addition, we could demonstrate that hAPLN was associated with more severe histopathological renal findings as well as with development of ESRD in long-term follow-up. Considering both previous findings and the present results, there are strong indications that patients with hAPLN belong to a high-risk SLE sub-group. Adequate follow-up and antihypertensive therapy are likely to prevent impairment of renal function, but this should ideally be studied in prospective studies. As a first step towards the performance of prospective treatment studies on patients with APLN, it is also important to reach an international consensus regarding the definition of APLN.

Associations between aPL and the HLA-DRB1*04 allele were reported be Galeazzi and colleagues [13]. We recently demonstrated that the HLA-DRB1*04 and HLADRB1*13 alleles alone and in particular the combination of these two alleles (HLA-DRB1*04/*13) were associated 
both with aPL of several specificities and with an increased risk of macrovascular events among SLE patients [12]. In this study, we extend these observations by demonstrating that microvascular lesions in the kidney are also associated with HLA-DRB1*13. Thus, a similar genetic factor may confer risk of aPL-associated disease in SLE.

The large consecutive sample size, also including repeated biopsies, gave us a unique opportunity to study the prevalence and natural history of hAPLN in SLE patients. Due to retrospective data collection, and lack of repeated measurements of aPL in some cases, we were, however, not able to fully define occurrence of APS.

Somewhat surprisingly, hAPLN patients had higher doses of prednisone at biopsy compared to LN individuals. This could be explained by the more severe phenotype with higher creatinine thus requiring more intense treatment, but the high steroid usage could also have contributed to the more pronounced hypertension seen. However, the more extensive use of corticosteroids, which is unlikely to affect hAPLN findings in general, point to the importance of performing renal biopsies in order to optimize treatment strategies and avoid overtreatment with corticosteroids and immunosuppressants.

Currently there are no consensus guidelines for treatment of APLN, but it is generally recommended that patients should receive anti-hypertensive and anticoagulative treatments. Considering the associated risk for impaired renal function, randomized trials are urgently needed to explore optimal treatment regimens in order to preserve renal function in this sub-group of SLE patients.

\section{Conclusion}

In conclusion, our results demonstrate that hAPLN is a severe, but not uncommon, finding in lupus patients with renal involvement. The association between renal pathology and occurrence of aPL indicates that hAPLN is a separate renal disease entity with different underlying pathogenic mechanisms compared to LN. Due to the increased risk of renal impairment, vasculopathies in LN require an increased awareness. Genetic findings may contribute to hAPLN.

\footnotetext{
Abbreviations

aCL: anticardiolipin antibodies; aPL: antiphospholipid antibodies; APLN: antiphospholipid-associated nephropathy; APS: antiphospholipid syndrome; $\beta_{2}$ GP1: $\beta_{2}$-glycoprotein-1; Cl: confidence interval; ELISA: enzymelinked immunosorbent assay; ESRD: end-stage renal disease; hALPN: histopathological antiphospholipid-associated nephropathy; HLA: human leukocyte antigen; Ig: immunoglobulin; LA: lupus anticoagulant; LN: lupus nephritis; OR: odds ratio; SLE: systemic lupus erythematosus; STAT4: signal transducer and activator of transcription factor 4; TMA: thrombotic microangiopathy.
}

\section{Competing interests}

The authors declare that they have no competing interests.

\section{Authors' contributions}

JG carried out data collection, statistical analysis, and interpretation of results, as well as drafting the manuscript. BS assessed the renal biopsies. AZ participated in data collection. LP participated in assessing the genetic data and interpreting the results. ES participated in the study design, statistical analysis, and interpretation of results, as well as helping to draft the manuscript. IG conceived the study and participated in the study design, data collection, interpretation of results, and drafting the manuscript. All authors critically revised the manuscript as well as reading and approving the final version prior to submission.

\section{Acknowledgements}

This study was supported by grants from the Swedish Research Council, Swedish Rheumatism Association, King Gustaf V's 80-year Foundation, Swedish Heart-Lung Foundation, Foundation in Memory of Clas Groschinsky, Stockholm County Council and Karolinska Institutet Foundations.

\section{Author details}

'Department of Medicine, Unit of Rheumatology, Karolinska University Hospital, Karolinska Institutet, S-171 76 Stockholm, Sweden. ${ }^{2}$ Department of Pathology and Cytology, Karolinska University Hospital, Karolinska Institutet, S-171 76 Stockholm, Sweden.

Received: 5 December 2014 Accepted: 31 March 2015

Published online: 27 April 2015

\section{References}

1. Vikerfors A, Johansson AB, Gustafsson JT, Jonsen A, Leonard D, Zickert A, et al. Clinical manifestations and anti-phospholipid antibodies in 712 patients with systemic lupus erythematosus: evaluation of two diagnostic assays. Rheumatology. 2013;52:501-9.

2. Petri M. Update on anti-phospholipid antibodies in SLE: the Hopkins' Lupus Cohort. Lupus. 2010;19:419-23.

3. Miyakis S, Lockshin MD, Atsumi T, Branch DW, Brey RL, Cervera R, et al. International consensus statement on an update of the classification criteria for definite antiphospholipid syndrome (APS). J Thromb Haemost. 2006;4:295-306

4. Tektonidou MG, Sotsiou F, Nakopoulou L, Vlachoyiannopoulos PG, Moutsopoulos HM. Antiphospholipid syndrome nephropathy in patients with systemic lupus erythematosus and antiphospholipid antibodies: prevalence, clinical associations, and long-term outcome. Arthritis Rheum. 2004;50:2569-79.

5. Zheng H, Chen Y, Ao W, Shen Y, Chen XW, Dai M, et al. Antiphospholipid antibody profiles in lupus nephritis with glomerular microthrombosis: a prospective study of 124 cases. Arthritis Res Ther. 2009;11:R93.

6. Daugas E, Nochy D, Huong DL, Duhaut $\mathrm{P}$, Beaufils $\mathrm{H}$, Caudwell V, et al. Antiphospholipid syndrome nephropathy in systemic lupus erythematosus. J Am Soc Nephrol. 2002;13:42-52.

7. Song D, Wu LH, Wang FM, Yang XW, Zhu D, Chen M, et al. The spectrum of renal thrombotic microangiopathy in lupus nephritis. Arthritis Res Ther. 2013;15:R12

8. Hughes GR. The antiphospholipid syndrome. Lupus. 1996;5:345-6.

9. McNeil HP, Hunt JE, Krilis SA. Antiphospholipid antibodies - new insights into their specificity and clinical importance. Scand J Immunol. 1992;36:647-52.

10. Galli M, Luciani D, Bertolini G, Barbui T. Lupus anticoagulants are stronger risk factors for thrombosis than anticardiolipin antibodies in the antiphospholipid syndrome: a systematic review of the literature. Blood. 2003;101:1827-32

11. Bolin K, Sandling JK, Zickert A, Jonsen A, Sjowall C, Svenungsson E, et al. Association of STAT4 polymorphism with severe renal insufficiency in lupus nephritis. PLoS One. 2013;8, e84450.

12. Lundstrom E, Gustafsson JT, Jonsen A, Leonard D, Zickert A, Elvin K, et al. HLA-DRB $1{ }^{*} 04 /{ }^{*} 13$ alleles are associated with vascular disease and antiphospholipid antibodies in systemic lupus erythematosus. Ann Rheum Dis. 2013;72:1018-25.

13. Galeazzi M, Sebastiani GD, Tincani A, Piette JC, Allegri F, Morozzi G, et al HLA class II alleles associations of anticardiolipin and anti-beta2GPI antibodies in a large series of European patients with systemic lupus erythematosus. Lupus. 2000;9:47-55.

14. Svenungsson E, Gustafsson J, Leonard D, Sandling J, Gunnarsson I, Nordmark G, et al. A STAT4 risk allele is associated with ischaemic 
cerebrovascular events and anti-phospholipid antibodies in systemic lupus erythematosus. Ann Rheum Dis. 2010;69:834-40

15. Tan EM, Cohen AS, Fries JF, Masi AT, McShane DJ, Rothfield NF, et al. The 1982 revised criteria for the classification of systemic lupus erythematosus. Arthritis Rheum. 1982;25:1271-7.

16. Churg JBJ, Glassock RJ. Renal Disease: Classification and Atlas of Glomerular Diseases. 2nd ed. New York: Igaky-Shoin; 1995.

17. Weening JJ, D'Agati VD, Schwartz MM, Seshan SV, Alpers CE, Appel GB, et al. The classification of glomerulonephritis in systemic lupus erythematosus revisited. Kidney Int. 2004;65:521-30.

18. Nochy D, Daugas E, Droz D, Beaufils H, Grunfeld JP, Piette JC, et al. The intrarenal vascular lesions associated with primary antiphospholipid syndrome. J Am Soc Nephrol. 1999;10:507-18.

19. Pengo V, Ruffatti A, Legnani C, Gresele P, Barcellona D, Erba N, et al. Clinical course of high-risk patients diagnosed with antiphospholipid syndrome. J Thromb Haemost. 2010;8:237-42.

20. Levey AS, Bosch JP, Lewis JB, Greene T, Rogers N, Roth D. A more accurate method to estimate glomerular filtration rate from serum creatinine: a new prediction equation, Modification of Diet in Renal Disease Study Group. Ann Intern Med. 1999;130:461-70.

21. Levey AS, Eckardt KU, Tsukamoto Y, Levin A, Coresh J, Rossert J, et al. Definition and classification of chronic kidney disease: a position statement from Kidney Disease: Improving Global Outcomes (KDIGO). Kidney Int. 2005;67:2089-100

22. Griffiths $M H$, Papadaki L, Neild GH. The renal pathology of primary antiphospholipid syndrome: a distinctive form of endothelial injury. QJM 2000;93:457-67.

23. Huong DL, Papo T, Beaufils H, Wechsler B, Bletry O, Baumelou A, et al. Renal involvement in systemic lupus erythematosus. A study of 180 patients from a single center. Medicine. 1999;78:148-66.

24. Gustafsson JT, Simard JF, Gunnarsson I, Elvin K, Lundberg IE, Hansson LO, et al. Risk factors for cardiovascular mortality in patients with systemic lupus erythematosus, a prospective cohort study. Arthritis Res Ther. 2012;14:R46.

25. Gustafsson J, Gunnarsson I, Borjesson O, Pettersson S, Moller S, Fei GZ, et al. Predictors of the first cardiovascular event in patients with systemic lupus erythematosus - a prospective cohort study. Arthritis Res Ther. 2009;11:R186.

\section{Submit your next manuscript to BioMed Central and take full advantage of:}

- Convenient online submission

- Thorough peer review

- No space constraints or color figure charges

- Immediate publication on acceptance

- Inclusion in PubMed, CAS, Scopus and Google Scholar

- Research which is freely available for redistribution 